\title{
EXACT PMF ESTIMATION OF SYSTEM INDICES IN BOUNDARY - CROSSING PROBLEM
}

\author{
ŞAHINNOĞLU M., GEBİZLIOĞLU Ö.L., \\ Department of Statisties, Middle East Technical University, Ankara, Turkey
}

\begin{abstract}
The objective of this research is to derive an exact probability mass function for the Bernoulli random variables indicating the quality of performance reliability at any instant of time in a discrete-time two-state maintainable-repairable physical system that fluctuates between operating and defective states; that is, above and below a fictitious level where zero level is the boundary between good and bad states in a Markovian or Nonmarkovian chain. Note that since these Bernoulli random variables do not necessarily possess identical success probabilities, we speak of nonstationary processes.
\end{abstract}

Given any discrete instant of time $n$ where the total length of study is $\mathbf{N}$ time units (hour, day, year), the discrete random variables of interest are defined as zero level $\left(O_{n}+\right.$ or $\left.O_{n}\right)$ and level-crossing $\left(Z_{n}=1\right.$ or $\left.Z_{n}=0\right)$. Hence provided that the probabilities of transitions from positive to negative $\left(P_{n}\right)$ and from positive to positive $\left(1-P_{n}\right)$ and from negative to positive $\left(P_{n} B\right)$ and from negative to negative $\left(1-P_{n} B\right)$ are specified earlier by sampling system data or simply given; the analyst can estimate the probability of being at negative state $P\left(O_{n^{-}}\right)=1-P\left(O_{n}+\right)$ and the probability of becoming defective $P\left(Z_{n}=1\right)=1-P\left(Z_{n}=0\right)$ given the system was operative at time increment $n-1$.

Further an analytical expression is derived for the two random variables of interest for general $n$ time increments with examples for up to $\mathbf{n}=5$. A simple digital Fortran written computer program evaluates the probabilities at a given $n$ for both random variables, hence completely specifying the probability mass function as illustrated in the tree-diagram. A system example is given to show the applicability of the algorithm with reference to both types of system, namely special and general case.

Key Words: Markov Chain, Bernoulli Process, Exact P.M.F.

\section{OBJECTIVES}

The objective is to derive an exact probability mass function, not an approximate or asymptotic $(1,2)$, for the reliability parameters at any instant of time to measure quality of performance in a discretetime two-state repairable-maintainable physical system that fluctuares between operating and defective, i.e. above and below a fictitious level, where zero level is the boundary between good and bad states as in a boundary-crossing scenario. Given any discrete instant

ISSN 0251-0871, A: Üniv. Basmevi 
of time $\mathbf{n}$ out of total $\mathrm{N}$ time units (hours, day, year), the discrete random variables of interest are defined as zero level $\left(0^{+}\right.$or $\left.0^{-}\right)$and level crossing $(Z=1$ or $Z=0)$ as in Graph 1 , Figure 1. Hence, provided the probabilities of transitions from positive to negative $\left(P_{n}\right)$ or from positive to positive $\left(1-P_{n}\right)$ and from negative to positive $\left(P_{n} B\right)$ or from negative to negative $\left(1-P_{n} B\right)$ are calculated earlier by sampling system data; the analyst wishes to estimate, in advance, the probability of being at positive state $P\left(O_{n}^{+}\right)$or negative state $P\left(O_{n}^{-}\right)$and the probability of becoming defective $P\left(Z_{n}=1\right)$ at time $n$, given the system was operative at $n-1$. The goal for system planner, for ex, in an electrical power generation system where positive may indicate excess energy reserve, is to meet any undesirable trends for certain time intervals, by taking preceutions such as adjusting for the total supply of power or production since the customer demand (load forecast) and chance failures can not be regulated by the analyst.

\section{METHODOLOGY}

Let, in electric power generation system as a boundary crossing problem, $m_{i}$ be the capacity (production) margins at $i=1,2, n ., . . N$ where $m_{i}$ $=$ INSCAP $-\mathrm{X}-\mathrm{L}_{\mathrm{i}}$ and INSCAP $=$ Installed Capacity (Production), $L_{i}=$ Load (Demand) Forecast at time $i$ independent of each other, $\mathrm{X}=$ Capacity Outage due to generator failure (production loss due to machine accident, etc.) whose p.d.f. is known. The r.v. zero level will be $\mathrm{O}^{+}$when margin is positive and $\mathrm{O}^{-}$when margin is negative. The r.v. zero crossing $Z$ will take on a value of unity if $m_{i}$ becomes negative given $m_{i-1}$ was positive, as below illustrated in Graph 1 which animates the boundary crossing problem. Then, a computer program in Fortran is written to generate Tables 1 and 2 in order to observe an imbedded trend following which formulae are derived in Tables 3 and 4 to possess the closed form probability mass functions for the discrete r.v.'s. The paper will conclude with an example and propositions developed for special and general case.

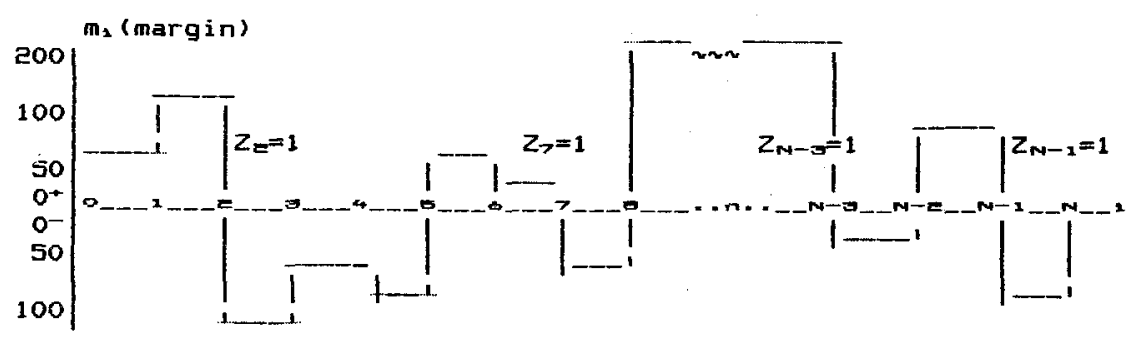

Graph 1. Illustration of the r. v.' $s: m, 0, \mathrm{Z}$ 
Following are the additive terms, referring to the tree-diagram in Fig. 1, to calculate the probability mass of the r.v. zero-level and zero-crossing respectively. These equations are under Table 1 and 2 .

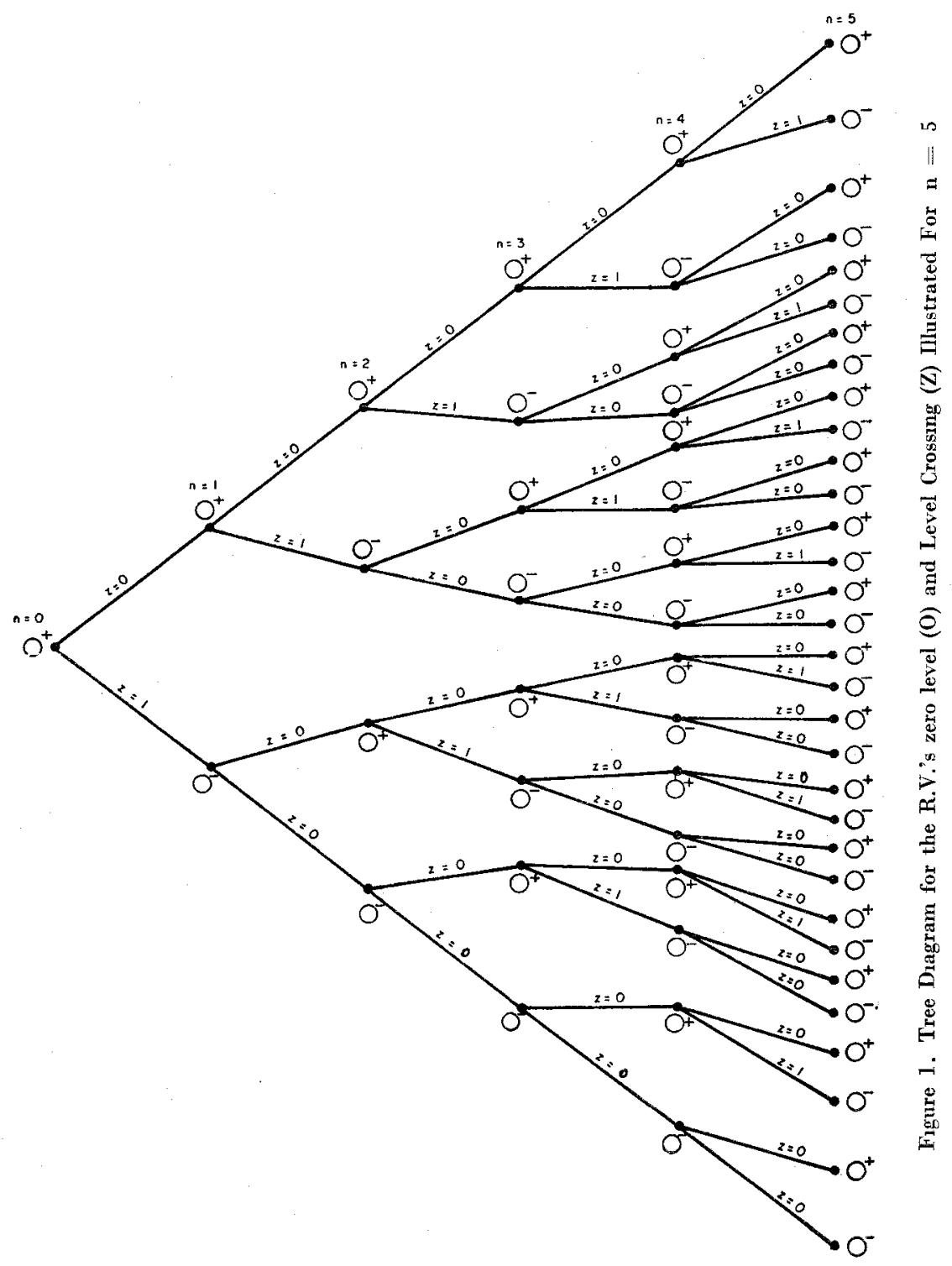




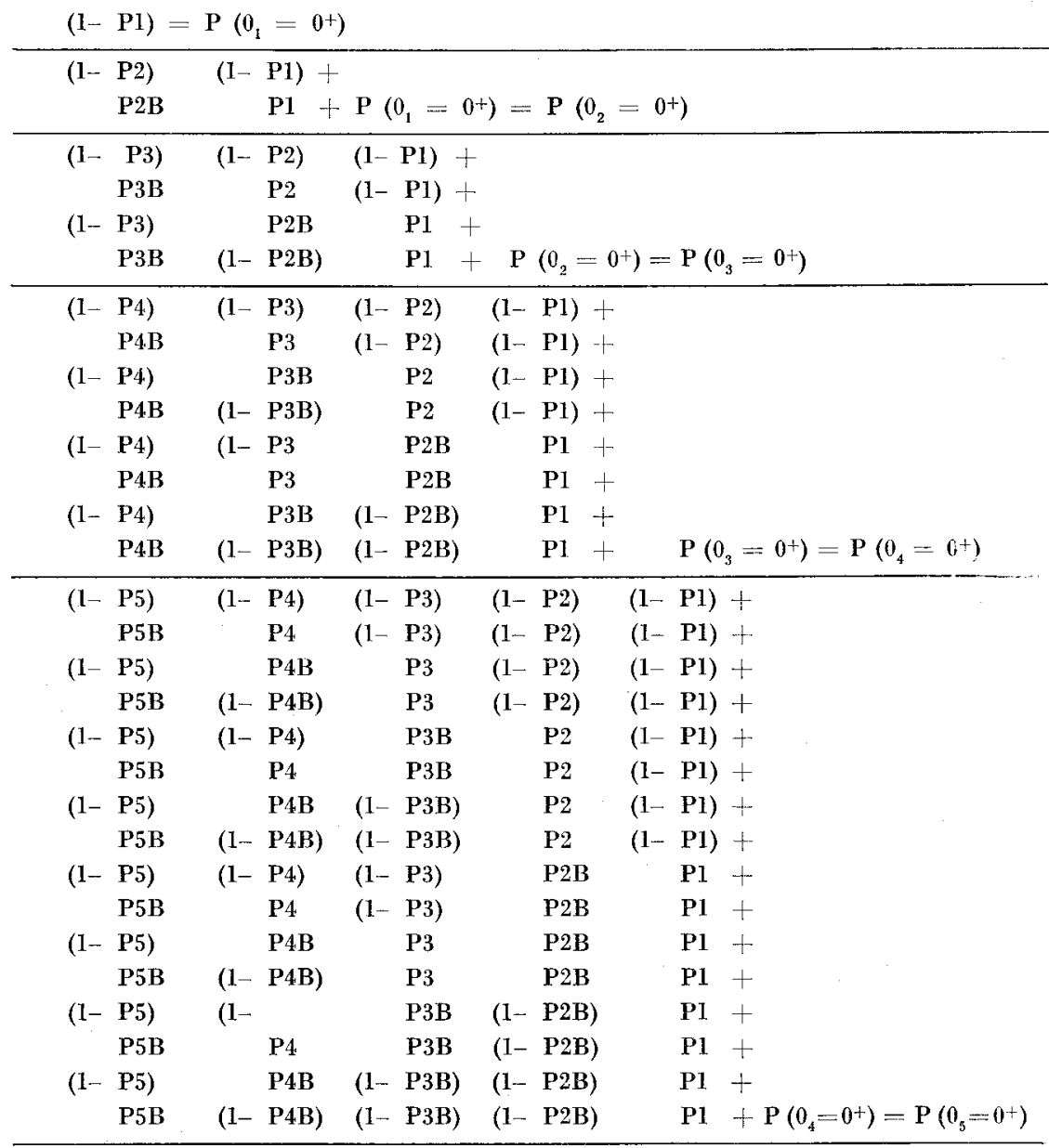

Table 2. Additive Terms For $\mathrm{P}\left(\mathrm{Z}_{5}=1\right)$

\begin{tabular}{lccc}
$\mathrm{P} 1$ & $=\mathrm{P}\left(\mathrm{Z}_{1}=1\right)$ \\
\hline P2 & $(1-\mathrm{P} 1)+\mathrm{P}\left(\mathrm{Z}_{1}=1\right)=\mathrm{P}\left(\mathrm{Z}_{2}=1\right)$ \\
\hline P3 & $(1-\mathrm{P} 2)$ & $(1-\mathrm{P} 1)+$ \\
P3 & P2B & P1 $+\mathrm{P}\left(\mathrm{Z}_{2}=1\right)=\mathrm{P}\left(\mathrm{Z}_{3}=1\right)$ \\
\hline P4 & $(1-$ P3 $)$ & $(1-\mathrm{P} 2)$ & $(1-\mathrm{P} 1)+$ \\
P4 & P3B & P2 & $(1-\mathrm{P} 1)+$ \\
P4 & $(1-$ P3 $)$ & P2B & P1 + \\
P4 & P3B & $(1-$ P2B $)$ & P1 $+\mathrm{P}\left(\mathrm{Z}_{3}=1\right)=\mathbf{P}\left(\mathrm{Z}_{4}=1\right)$ \\
\hline
\end{tabular}




\begin{tabular}{rcrrr} 
P5 & $(1-$ P4 & $(1-$ P3 & $(1-$ P2 $)$ & $(1-$ P1 + \\
P5 & P4B & P3 & $(1-$ P2 $)$ & $(1-$ P1 + \\
P5 & $(1-$ P4 $)$ & P3B & P2 & $(1-$ P1 + \\
P5 & P4B & $(1-$ P3B $)$ & P2 & $(1-$ P1 + \\
P5 & $(1-$ P4) & $(1-$ P3 $)$ & P2B & P1 + \\
P5 & P4B & P3 & P2B & P1 + \\
P5 & $(1-$ P4 $)$ & P3B & $(1-$ P2B $)$ & P1 + \\
P5 & P4B & $(1-$ P3B $)$ & $(1-$ P2B $)$ & P1 + P $\left(Z_{4}=1\right)=\mathbf{P}\left(Z_{5}=1\right)$ \\
\hline
\end{tabular}

The above computer layout can as well be put into an analytical expression for general " $n$ " as in the following equations illustrated as in Table 3 and 4.

Table 3. P.M.F. For The Zero Level R.V. Where Where $P\left(0_{n}-\right)=1-P\left(0_{n}{ }^{+}\right)$

$$
\begin{aligned}
& \mathbf{P}\left(0_{n}+\right)=\stackrel{n}{\pi}\left(1-P_{i}\right)+\bar{P}_{n} P_{n-i}^{n-2} \pi\left(1-P_{i}\right)+\left(1-P_{n}\right) \bar{P}_{n-1}^{-} P_{n-2}^{n-3} \pi\left(1-P_{i}\right)+\bar{P}_{n}^{-}\left(1-\ddot{P}_{n-1}\right)
\end{aligned}
$$

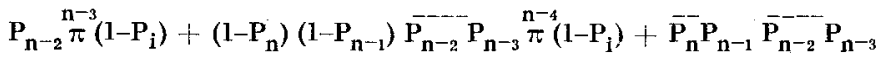

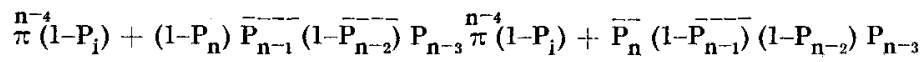

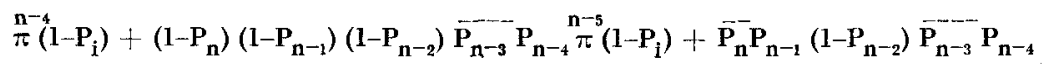

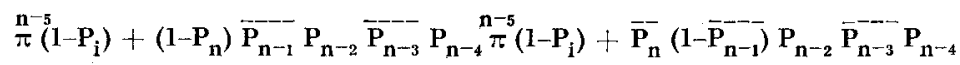

$$
\begin{aligned}
& { }_{\pi\left(1-P_{1}\right.}^{n-5}+\left(1-P_{n}\right)\left(1-P_{n-1}\right) \widetilde{P_{n-2}}\left(1-\bar{P}_{n-3}^{-}\right) P_{n-4}^{n-5} \pi\left(1-P_{i}\right)+\bar{P}_{n} P_{n-1} \overline{P_{n-2}^{-}}\left(1-\bar{P}_{n-3}^{--}\right) P_{n-4} \\
& \pi\left(1-P_{i}\right)+\left(1-P_{n}\right) \overline{P_{n-1}^{--}}\left(1-\bar{P}_{n-2}^{--}\right)\left(1-\bar{P}_{n-3}^{-}\right) P_{n-4}^{n-5} \pi\left(1-P_{1}\right)+\bar{P}_{n}^{-}\left(1-\overline{P_{n-1}^{-}}\right)\left(1-\overline{P_{n-2}}\right)\left(1-\overline{P_{n-3}^{-}}\right) \\
& P_{n-4}{ }_{n-5}^{n-5}\left(1-P_{i}\right)+\left(1-P_{n}\right)\left(1-P_{n-1}\right)\left(I-P_{n-2}\right)\left(1-P_{n-3}\right) \bar{P}_{n-4}^{--} P_{n-5}^{n-6} \pi\left(1-P_{i}\right)+\bar{P}_{n}^{-} P_{n-1} \\
& \left(1-P_{n-2}\right)\left(1-P_{n-3}\right) \bar{P}_{n-4}^{---} P_{n-5}^{n-6} \pi\left(1-P_{i}\right)
\end{aligned}
$$

\begin{tabular}{|c|}
\hline 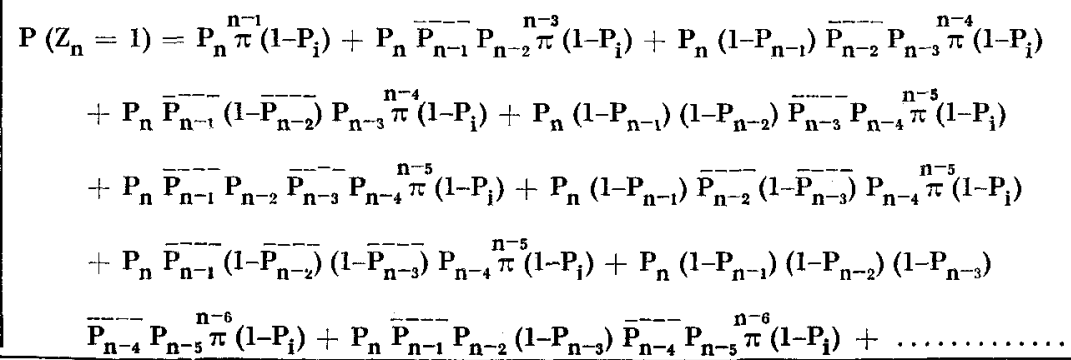 \\
\hline
\end{tabular}

Table 4. P.M.F. For The Zero Crossing R.V. Where $P\left(Z_{n}=1\right)=1-P\left(Z_{n}=0\right)$ 
The formulae for the e.m.f. of the r.v.'s of interest are derived for general $n$ thanks to the trend inherent in the Tables 1 and 2. Note that a product symbol $\pi$ with negative superscript multiplies the rest by zero, unity superscript multiplies by one. All products start with $\mathrm{i}=1$. Same numerical results are obtained through analytical calculation and the computer program that generated Tables 1 and 2 . Before studying a test system and developing a proposition, it is worth mentioning what is meant by special and general cases: If in the system described as above, at any increment of time $n, 1-P_{n}=P_{n} B, B$ : Bar; one minus probability of system malfunction while operating is equal to probability of system repair. then it is special; else general.

\section{APPLICATION AND CONCLUSION}

An IEEE Reliability Test System (Vol. PAS-100, May' 81, p. 2328 ) is studied with only the first six hours of load data for simplicity. Initially, a discrete probability table for $X=$ Capacity Outage, is found so that at each $\mathrm{i}: \mathbf{P}_{\mathbf{i}}=\mathbf{P}\left(\mathbf{m}_{\mathrm{i}}<0\right)=\mathbf{P}_{\mathbf{i}}\left[\left(\mathrm{X} \mid 0^{+}\right)>\right.$INSCAP $\left.-\mathrm{L}_{\mathrm{i}}\right]$. Note that in special-case, $\mathrm{P}_{\mathrm{i}} \mathrm{B}$ are equal to $\mathrm{I}-\mathrm{P}_{\mathrm{i}}$ and $\mathrm{P}\left[\mathrm{X}\left(\mathrm{O}^{+}\right)\right.$ $>$ Reserve $\left._{i}\right]$ is same as $\mathrm{P}\left[\left(\mathrm{X} \mid \mathrm{O}^{-}\right)>\right.$Reserve $\left._{i}\right]$. However, if otherwise for the general-case; the probability of outage exceeding reserve given at positive margin is not necessarily equal to that of given at negative margin. Hence, the system so behaves that the probability of malfunction while at positive state is not necessarily identical to the probability of continuing to malfunction while residing at negative state. An analog in stock market is that the probability of a stock price falling below threshold level while above is not same as the probability of keeping below once below.

In computing the indices unavilability $\mathrm{P}\left(\mathrm{O}_{\mathrm{n}}{ }^{-}\right)$and zero-crossing $P\left(Z_{n}=1\right)$; the $P_{i}$ and $P_{i} B$ are fed in order except for $P_{1} B$, as systems are planned to start with positive margin. For IEEE Example $\mathbf{n}=6$ above, the data read as follows: .031230, .095529, .904471, .059232, $.946768, .046437, .953563, .039648, .960352, .000728, .999272$ for special-case.

In general-case, given: $.031230 \quad .095529, \quad .904471, \quad .059232$, $.946768, .046431, .953563, .039648, .80000, .000728, .90000$ are the input data.

The output is, for special and general cases, respectively: $P\left(0_{6}{ }^{-}\right)$ $=.000728, \quad \mathrm{P}\left(\mathrm{Z}_{6}=1\right)=.00069914 ; \quad \mathrm{P}\left(0_{6}{ }^{-}\right)=.00540314, \mathrm{P}\left(\mathrm{Z}_{6}=\right.$ 
$1)=.00069372$. The trends inherent for special-case are: i) the probability of being in the negative state at $n$ is equal to the probability of margin less than zero at $n$ regardless of the past, ii) the probability of zero crossing at $n$ is equal to the product of being in the positive state at $n-1$ and probability of margin less than zero at $n$. For general case, use the recursive formulae or a computer program with recursive 'do loops'.

Proposition 1. In two-state (good or bad), discrete-time $\mathrm{i}=1$, $\mathrm{n}, \ldots, \ldots \mathrm{N}$ and maintainable-repairable (with forgetfulness property) physical system; the probability of being unavailable or in the bad (unwanted) state at $n$ is simply identical to the $P_{n}$, the probability of experiencing a zero crossing is equal to product of $P\left(0_{n-1}{ }^{+}\right)$and $P\left(0_{n}\right)$ for special system.

Proposition 2. Otherwise, for general systems where for all $i=1$, $n, \ldots, N, P_{i}$ is not equal to $1-P_{i} B ; P\left(O_{n}^{-}\right)$and $P\left(Z_{n}=1\right)$ can be calculated in the like manner to Tables 1 and 3 and formulae in Tables 2 and 4.

This study determines an exact p.m.f. of random variables, critical in decision making, for discrete-time, 2-state, maintainable-repairable system where the r.v.'s are assumed neither independent nor identical. It is illustrated that by clear time sequence representation of such a system process, dependency relation of zero level and level crossing variables can be specified in terms of imbedded conditional probabilities, found as result of prior system sampling and data analysis. This leads to determination of exact probability functions of said r.v.'s at any given time point irrespective of how large it is. Asymptotical theory approaches in the same respect face with difficulties especially in the case of evolutionary systems with dependent variables $[3,4]$. This study may further shed light on the nature of convergence in the related functional spaces to allow comparison of asymptotic and exact results. Further, this study can certainly be extended to multi-state systems.

\section{REFERENCES}

ŞAHİNOĞLU M., LONGNECKER M.T., RINGER L.J., SINGH C., AYOUB A.K., 1983. Pro* bability distribution functions for generation reliability indices-analytical approachIEEE Pas Vol. 102, p. 1486-93,

ŞAHİNOĞLU M. 1985. Probability distribution functions for generation reliability indices-analytical approach II- IMSIBAC III, Bilbao,

SERFLING R.J. 1968. Contributions to central limit theory for dependent variables, Annals of Math. Stat. Vol. 39, No. 4, p. 1158-75.

WANG. Y.H. 1981. On the limit of the Markov Binomial Distribution, J. App. Prob., 18, 937-942. 